\title{
Assessment of Left Ventricular Diastolic Function by Doppler Echocardiography
}

\author{
Michael Y Henein ${ }^{1,2}$ and Per Lindqvist ${ }^{1}$ \\ 1. Departments of Public Health and Clinical Medicine and Surgery and Perioperative Sciences, Umea University, Umea, Sweden and \\ 2. Canterbury Christ Church University, Kent, UK
}

\begin{abstract}
Diastole is an essential part of the cardiac cycle, during which significant changes in myocardial function, ion and energy transfer, as well as coronary flow, occur. In contrast to systole, diastole consists of four phases, each of which has its distinguishing function and events. However, the four phases are inter-related with events in early diastole correlating with those in late diastole and those occurring during the isovolumic relaxation time predicting both. The complexity of diastolic phases is reflected in the ways by which diastolic function is assessed. While intra-cardiac flow velocities, into and out of the atria, are measured by pulsed-wave Doppler, those of wall motion are assessed by M-mode, myocardial Doppler velocities or, recently, speckle tracking technique. Optimum integration of various aspects of diastolic function should always be considered in order to obtain an accurate comprehensive assessment, bearing in mind factors that normally affect it, for example age.
\end{abstract}

\section{Keywords}

Diastole, myocardial doppler velocities, filling velocities, LV diastolic function, left atrial function

Disclosure: The authors have no conflicts of interest to declare.

Received: 7 July 2015 Accepted: 4 August 2015 Citation: Cardiac Failure Review, 2015;1(2):87-9

Correspondence: Professor Michael Y Henein, Department of Public Health and Clinical Medicine, Umea University, Umea, Sweden SE-901 87.

E: michael.henein@umu.se

Diastole is an important period in the cardiac cycle when all heart components integrate together to secure optimum ventricular filling which determines the stroke volume pumped by the ventricle in the succeeding cycle. Three diastolic phases are well-recognised; early diastole, diastasis and late diastole 'atrial systole' (see Figure 1). To describe the actual events happening in the ventricle the three phases are named; early filling, diastasis and late filling.

Such description also reflects events happening between the time of mitral valve opening and closure. However, studies have already shown that patterns of diastolic events are determined by the duration and the extent of left ventricular shape change happening during the isovolumic relaxation time, with abnormal prolongation reflecting slow ventricular relaxation and abnormal shortening identifying raised left ventricular (LV) end-diastolic pressure and consequently left atrial pressure.1.2

Similar extreme patterns are often seen in patients with heart failure, but not exclusively so. While heart failure is a clinical diagnosis, it reflects various severities of cardiac function disturbances; hence there is no single diastolic pattern that is characteristic for a clinical diagnosis of heart failure. Other factors have been shown to influence diastolic function and these should be clearly understood before heart failure is discussed.

\section{Age and Diastolic Function}

With age, significant changes occur in diastolic function over and above those affecting systolic function. In the young up to the fourth decade of life, the left ventricle fills with a dominant early diastolic volume followed by a smaller late diastolic volume. The pronounced early diastolic phase is caused by the low (negative) apical pressures compared with those at the base of the LV, maintained by an apical untwist, anticlockwise rotation of the cardiac apex, (see Figure $1 A-C$ ) which results in a suction effect. With progressive collagen deposition in the myocardium, in the fifth decade of life, its relaxation slows and is delayed. This results in prolongation of isovolumic relaxation time due to delayed opening of the mitral valve. This occurs in the overall diastolic period but especially in the early diastolic phase (see Figure 1B), and results in compromised early filling component (or volume) with a compensatory increase in the late diastolic component and a more pronounced apical untwist (see Figure 1). ${ }^{3}$ These changes are greater if there is additional pathology affecting the left ventricle e.g. coronary artery disease or systemic hypertension. In the worst cases, the early filling can be completely truncated and the left ventricle fills with an isolated late diastolic filling component. If such patients develop atrial fibrillation, the isolated late diastolic filling component will be shifted to early diastole but at the expense of raising the left atrial pressure and reducing the stroke volume. ${ }^{4,5}$

\section{Electric Function and Diastole}

Diastolic phases are also influenced by the electrical pattern of activation (depolarisation) and repolarisation. QRS broadening, irrespective of bundle branch block (BBB) is associated with delayed activation and delayed inward motion. This is reflected in delayed segmental outward motion with post-ejection shortening, the combination of the two results in delayed onset and shortened early diastolic filling of the left ventricle. ${ }^{6}$ The same pattern is seen in coronary artery disease, particularly in 

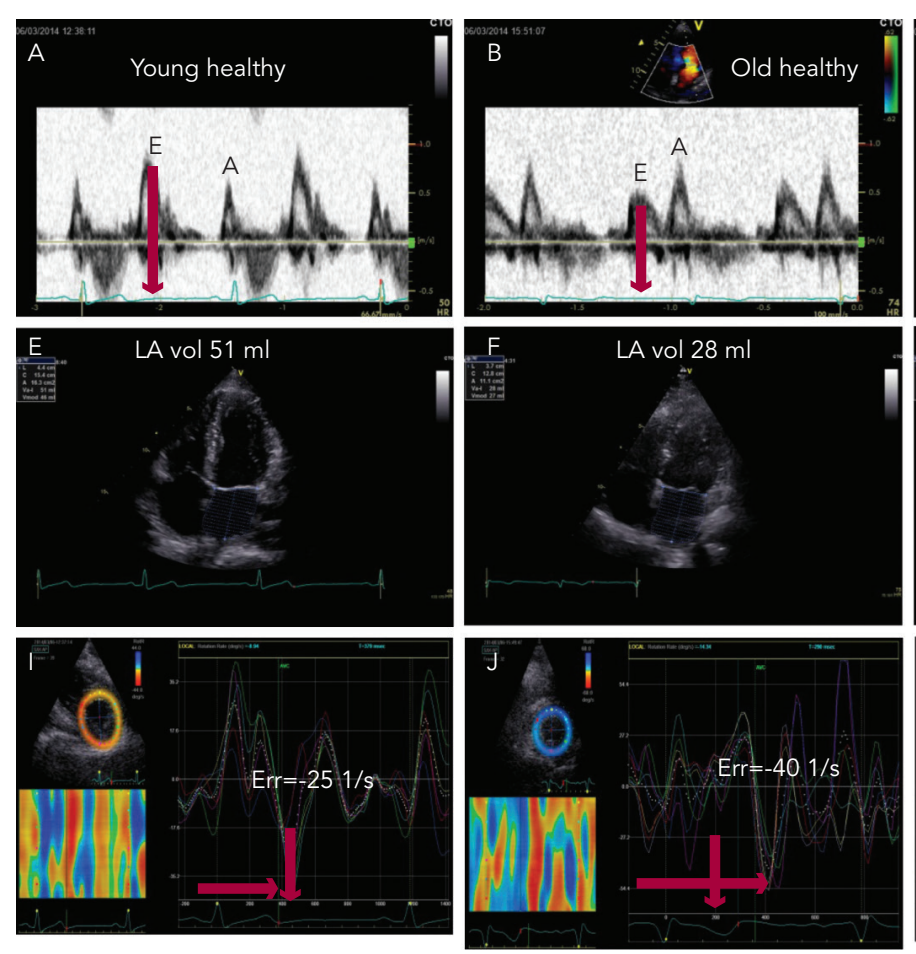

patients with Q wave infarction.7 Left ventricular filling pattern could also be affected by other electric abnormalities. An absent $P$ wave (e.g. through atrial fibrillation) results in absent late diastolic filling component, which compromises the overall filling and stroke volume of the ventricle. ${ }^{8,9}$

The most sensitive myocardial layer to coronary artery disease and ischaemia is the subendocardium, even in patients with no obstructive lesions in the epicardial vessels. Indeed, early subendocardial dysfunction has been shown to be predominantly diastolic and as it worsens it becomes systolic. Disturbances are similar to those described above but the delayed segmental shortening and lengthening is more profound..$^{10}$ These disturbances will affect the LV filling pattern as previously described."

In patients with scarred myocardium from a prior infarction, the overall function of that segment might be dyssynchronous, hence adding to the extent of filling abnormalities. In severe cases and with more than one dyssynchronous segment the left ventricle might fill with an isolated late diastolic component. ${ }^{10,12}$

\section{Diastolic Patterns and Heart Failure Symptoms}

The main symptom of heart failure is breathlessness. If cardiac in origin it is likely to be caused by either impaired emptying of the left atrium due to early diastolic dysfunction (relaxation disturbances) or due to raised left atrial pressure (restrictive filling pattern) as a complication to raised LV end-diastolic pressure. The latter has clear characteristics; dominant early diastolic component, short isovolumic relaxation time (see Figure $1 C$ and $D$ ), reduced apical early diastolic untwist rate ${ }^{13}$ and increased duration of flow reversal in the pulmonary veins in late diastole. The two conditions result in left atrial enlargement, reduced compliance and unstable function, hence potential development of atrial arrhythmia and even fibrillation (see Figures $1 G$ and $H$ )..$^{11,14}$
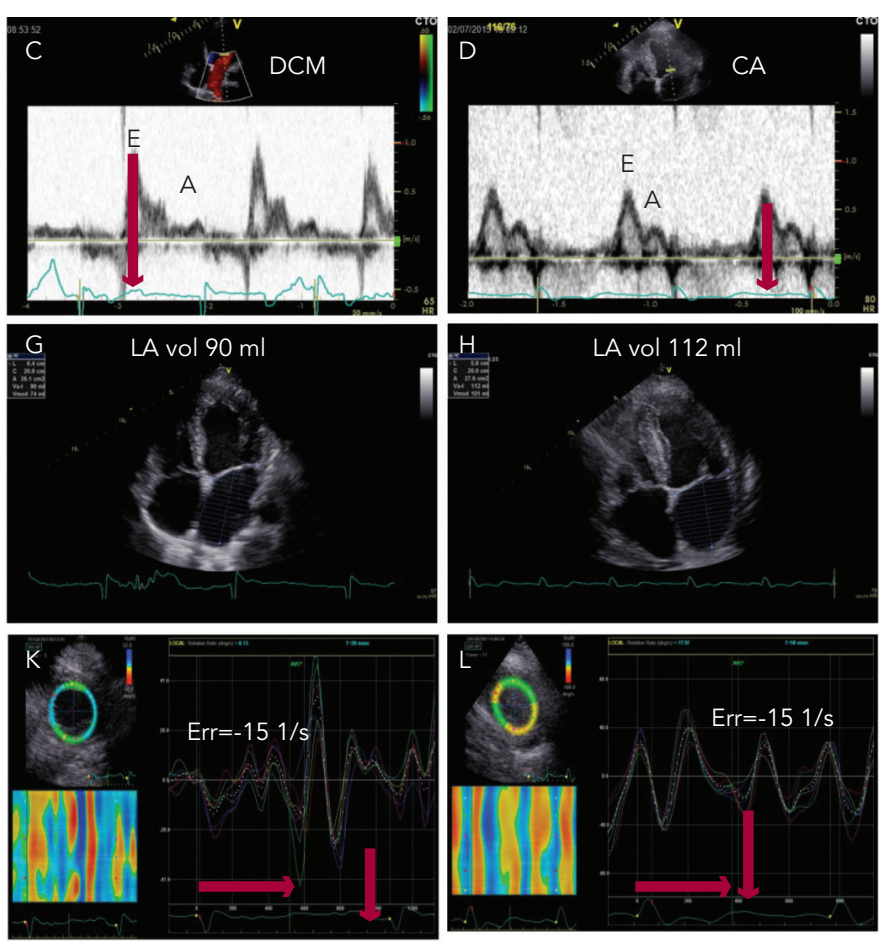

Diastolic Disturbances and Heart Failure Signs

A third heart sound heard in heart failure is consistent with restrictive LV filling and is caused because of rapid change of early diastolic filling acceleration to deceleration. This condition reflects unstable cardiac function that needs left atrial pressure off-loading therapy with vasodilators. A fourth sound reflects isolated late diastolic filling due to severe early diastolic dyssynchrony. Signs of pulmonary oedema reflect raised left atrial (LA) pressure, which is secondary to stiff $L V$ and raised end-diastolic pressure. Most patients with this condition present with some degree of mitral regurgitation, which further accentuates LA pressure. Systemic fluid retention is a sign of either stiff right ventricle (RV) or significant tricuspid regurgitation, secondary to left heart disturbances or due to primary right heart problems with or without pulmonary hypertension. ${ }^{15,16}$

\section{Measurements of Diastolic Function}

Doppler echocardiography in the assessment of diastolic function has a number of limitations; mainly relating to heart rate and arrhythmias, in addition to the ones mentioned above. Therefore, when assessing diastolic function the general recommendation is to use more than one measurement. LV filling volumes are studied by Doppler velocities obtained from the transmitral valve flow velocities in early diastole (E) and during atrial systole (A) waves. While peak velocities reflect diastolic function well, additional measurements should be considered to avoid any potential overor underestimation. E wave deceleration time is a good marker of raised LV end-diastolic pressure. A short isovolumic relaxation time - the interval between the end of the aortic Doppler velocity signal and the onset of the E wave - is a good reflection of LA pressure changes, although not in absolute terms. Also, a simple observation of E:A ratio could give a rough impression of diastolic function. In a patient aged over 50 , a normal filling pattern is expected to have an $\mathrm{E}: \mathrm{A}<1.0$, in the presence of normal LA size $\left(<28 \mathrm{ml} / \mathrm{m}^{2}\right)$, normal LV configuration and normal LV intrinsic relaxation. With increasing left 
atrial pressures, the combination of $E: A>1$, enlarged $L A$, abnormal LV function and abnormal LV intrinsic relaxation (such as reduced $\left.e^{\prime}\right)$ is expected ${ }^{17,18}$.

\section{Relaxation Abnormality}

This condition is mainly characterised by reduced $E$ wave velocity, increased $A$ wave velocity, E:A $<0.8$, prolonged $E$ wave deceleration time (>200 ms) and isovolumic relaxation time (IVRT $>100 \mathrm{~ms}$ ). This LV filling pattern is also commonly seen in the healthy elderly population aged above 60 years and therefore has low accuracy in detecting heart failure (see Figure 1B). However, if this pattern is present in conjunction with enlarged LA (volume $>34 \mathrm{ml} / \mathrm{m}^{2}$ ) a strong suspicion of unstable LA pressures is raised and exercise provocation can be useful to detect further increases in LA pressure as shown by changes in E:A.19

\section{Restrictive LV Filling Pattern}

This condition is easily detected in heart failure with $E: A>2$, short $E$ wave deceleration time ( $<150 \mathrm{~ms}$ ) and IVRT ( $<60 \mathrm{~ms})$. The majority of these patients also have increased LA volume $\left(34 \mathrm{ml} / \mathrm{m}^{2}\right)$ and some degree of raised pulmonary artery pressures. This pattern is commonly found in newly diagnosed patients or those not responding to optimum medical therapy because of immobile LV myocardium. Such pattern of cardiac function reflects instability that needs aggressive LA pressure offloading treatment with vasodilators.

In between the abnormal relaxation pattern and restrictive pattern there is a wide range of diastolic patterns but all could be described in one category based on the following measurements; LA volume, E:A, IVRT and DT etc. In those patients new additional methods have been proposed that help optimum evaluation e.g. $e^{\prime}$ from tissue Doppler echocardiography and calculating $E / \mathrm{e}^{\prime}$, time difference between the duration of the transmitral $A$ wave and respective retrograde pulmonary venous $\operatorname{Ar}(\mathrm{Ar}-\mathrm{A})$. An E/e' (mean of septal and lateral LV e') $>13$ and Ar-A $>30$ ms are highly indicative of elevated LA pressure. ${ }^{19}$

\section{Filling Pattern in Atrial Fibrillation}

Irrespective of LV size and function, the development of myocardial stiffness and the rise in LV diastolic pressures cause LA enlargement, which with time may become unstable and cause fibrillation. In these patients there is a single component of LV filling, the E wave, with absent A wave on the transmitral and pulmonary venous flow, hence, E:A ratio and A wave duration difference are not applicable. However, $E / e^{\prime}$ remains of diagnostic value for raised LA pressure in addition to the short $E$ wave deceleration and the increased LA volume. ${ }^{20}$

\section{Diastolic Function Measurements in HFpEF}

Heart failure with preserved ejection fraction (HFpEF) has no specific characteristic pattern of diastolic function. All the patterns described above apply to HFpEF, in particular the presence of LA enlargement. In addition, an E:A >1.5 in an elderly patient is highly suggestive of elevated filling pressures. The only difference is the disproportionately small volume of the LA with respect to the poor LV myocardial function in patients with amyloid heart disease, in whom the left atrium could be enlarged only to a small degree

\section{Diastolic Function in Fast Heart Rate}

It is very important not to apply the absolute values of diastolic function parameters in patients with heart failure when presenting with tachycardia. Fast heart rate affects diastole before systole, hence all diastolic intervals and values are changed. Diastasis becomes minimal and the two filling components grow closer until they eventually merge and become one summation filling component. Therefore, the best way to assess diastolic function in this scenario is by correcting all time intervals to heart rate. An example of such a method is the assessment of total isovolumic time, which is the product of subtracting the sum of the filling time and ejection time from R-R interval and correcting it for heart rate. This gives a value in $\mathrm{ms} / \mathrm{s}$. It is simple and highly reproducible. It has also been shown to be very accurate in assessing the presence and severity of LV dyssynchrony in heart failure. Furthermore, the LV filling time can be measured and indexed to RR and normally it should not decrease by more than $40 \%$ at peak exercise.

\section{Diastolic Function and Ventricular Interaction}

Ventricular interactions are known as a haemodynamic phenomenon from the studies by Bernheim. ${ }^{21}$ However, in primary RV disease, such as pressure overload (pre-capillary PH) or RV volume overload, the overload creates a septal shift as well as low LV filling volume. commonly in both conditions a reduced LV E:A could be expected, and this has been shown to have a significant prognostic value. ${ }^{22}$
1. Henein MY, Gibson DG. Abnormal subendocardial function in restrictive left ventricular disease. Br Heart J 1994;72:237-42.

2. Brecker SI, Lee CH, Gibson DG. Relation of left ventricular isovolumic relaxation time and incoordination to transmitral Doppler filling patterns. Br Heart J 1992;68:567-73.

3. Phan TT, Shivu GN, Abozguia K, et al. Left ventricular torsion and strain patterns in heart failure with normal ejection fraction are similar to age-related changes. Eur J Echocardiog 2009; 10:793-800

4. Henein $M$, Lindqvist $P$, Francis $D$, et al. Tissue Doppler analysis of age-dependency in diastolic ventricular behaviour and filling: a cross-sectional study of healthy hearts (the Umea General Population Heart Study). Eur Heart 2002;23:162-71.

5. Bukachi F, Waldenstrom A, Morner S, et al. Age dependency in the timing of mitral annular motion in relation to ventricular filling in healthy subjects: Umea Genera Population Heart Study. Eur J Echocardiogr 2008;9:522-9.

6. Xiao HB, Brecker SJ, Gibson DG. Effects of abnormal. activation on the time course of the left ventricular pressure pulse in dilated cardiomyopathy. Br Heart 1992;68:403-7.

7. O'Sullivan CA, Ramzy IS, Duncan A, et al. The effect of the $Q$ wave infarct on left ventricular electromechanical function. Int J Cardiol 2003;92:71-6.
8. O'Sullivan CA, Ramzy IS, Li W, et al. The effect of the localisation of $\mathrm{Q}$ wave myocardial infarction on ventricular electromechanics. Int J Cardiol.2002;84:241-7.

. Xiao HB, Brecker SJ, Gibson DG. Differing effects of right ventricular pacing and left bundle branch block on left ventricular function. Br Heart J 1993;69:166-73.

10. Henein MY, Priestley K, Davarashvili T, et al. Early changes in left ventricular subendocardial function after successful coronary angioplasty. Br Heart J 1993;69:501-6.

11. Henein MY, Gibson DG. Suppression of left ventricular early diastolic filling by long axis asynchrony. Br Heart J 1995; $73: 151-7$

12. Henein MY, O'Sullivan C, Davies SW, et al. Effects of acute coronary occlusion and previous ischaemic injury on left ventricular wall motion in humans. Heart 1997;77:338-45.

13. Omar AM, Vallabhajosyula S, Sengupta PP. Left ventricular twist and torsion: research observations and clinical applications. Circ Cardiovasc Imaging 2015;8:e003029.

14. Duncan AM, Lim E, Gibson DG, Henein MY. Effect of dobutamine stress on left ventricular filling in ischaemic dilated cardiomyopathy: pathophysiology and prognostic implications. J Am Coll Cardiol 2005;46:488-96.

15. Henein MY, Amadi A, O'Sullivan C, et al. ACE inhibitors unmask incoordinate diastolic wall motion in restrictive left ventricular disease. Heart 1996:76:326-31.
16. Henein MY, O'Sullivan CA, Coats AJ, Gibson DG. Angiotensinconverting enzyme (ACE) inhibitors revert abnormal right ventricular filling in patients with restrictive left ventricular disease. I Am Coll Cardiol 1998;32:1187-93.

17. Nagueh SF. Noninvasive estimation of LV filling pressures in heart failure and reduced ejection fraction: revisited and verified. JACC Cardiovasc Imaging 2011;:4:935-7.

18. Nagueh SF, Middleton KJ, Kopelen HA, Zoghbi WA, Quinones MA. Doppler tissue imaging: a noninvasive technique for evaluation of left ventricular relaxation and estimation of filling pressures. J Am Coll Cardiol 1997;30:1527-33.

19. Nagueh SF, Appleton CP, Gillebert TC, et al. Recommendations for the evaluation of left ventricular diastolic function by echocardiography. J Am SOC Echocardiog 2009;22:107-33.

20. Nagueh SF, Kopelen HA, Quinones MA. Assessment of left ventricular filling pressures by Doppler in the presence of atrial fibrillation. Circulation 1996;94:2138-45.

21. Henein MY, Xiao HB, Brecker SJ, Gibson DG. Berheim "a" wave: obstructed right ventricular inflow or atrial cross talk? Br Heart J 1993;69:409-13.

22. Henein MY, Gronlund $\mathrm{C}$, Tossavainen $\mathrm{E}$, et al. Right and left heart dysfunction predict mortality in pulmonary hypertension. Clin Physiol Funct Imaging 2015: epub ahead of press. 